\title{
Transjugular intrahepatic portosystemic shunt in Budd-Chiari syndrome
}

\author{
Sajad Ahmad Salati ${ }^{\mathrm{a},}$
}

${ }^{a}$ Associate Professor of Surgery, The Unaizah College of Medicine and Applied Medical Sciences, Qassim University, KSA.

\section{DEAR SIR}

A 29-year-old female who was a known case of primary Budd-Chiari Syndrome (BCS) reported with nonspecific abdominal discomfort. She had a history of multiple variceal bleeds and had undergone placement of a transjugular intrahepatic porto-systemic shunt (TIPS) three months before this episode. She was on a follow-up regimen at a regional tertiary care center for hepatic disorders. The patient was managed conservatively because the hemodynamic and laboratory parameters were within acceptable limits. Imaging

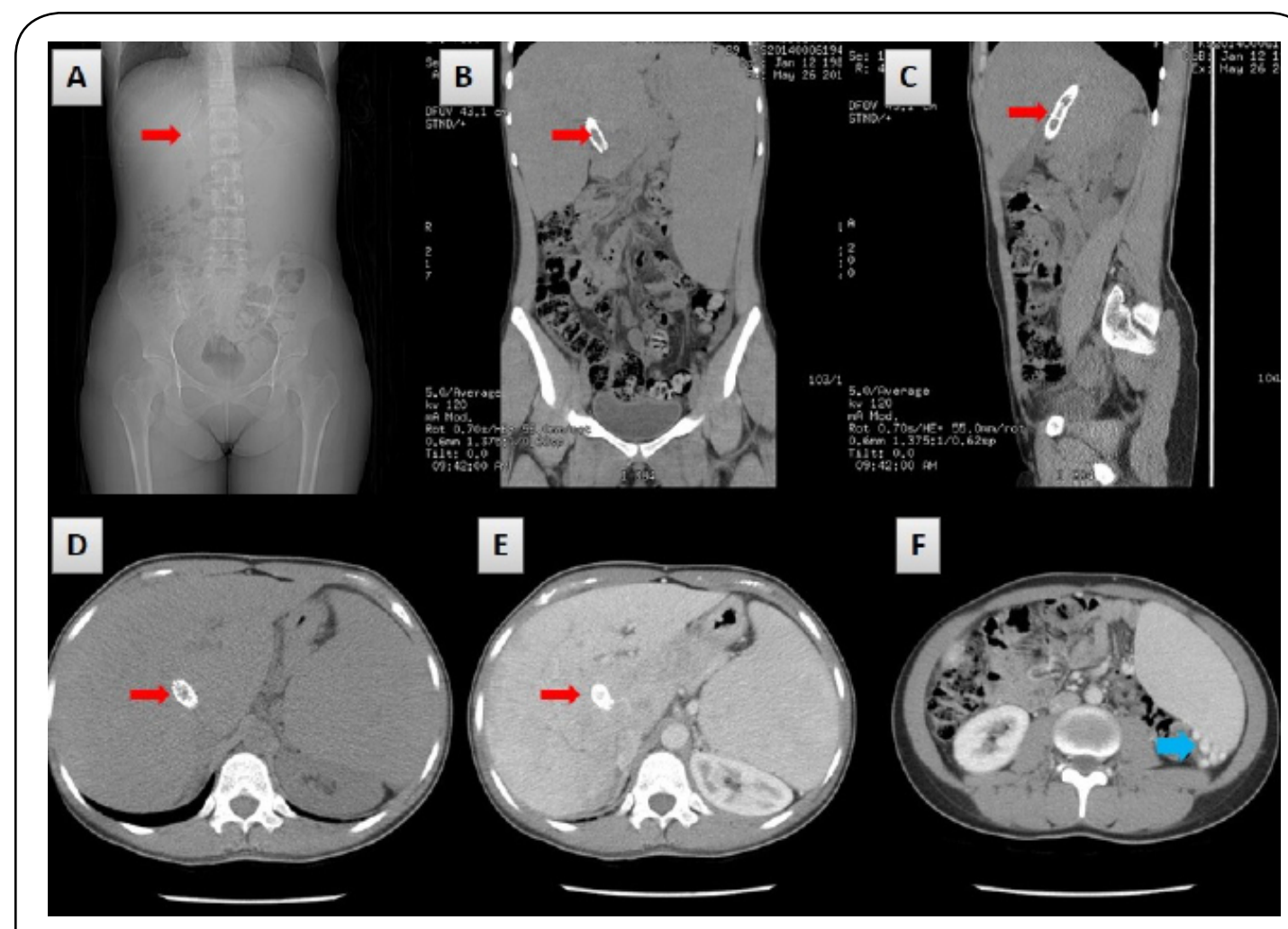

Figure 1. CT Scan showing patent TIPS (red arrows) and dilated collaterals (blue arrow). (A) Scout film. (B) Coronal view. (C) Sagittal view. (D) Transverse section (without contrast). (E,F) Transverse section (with contrast).

* Corresponding author: Sajad Ahmad Salati

Mailing address: Associate Professor of Surgery, The Unaizah College of Medicine and Applied Medical Sciences, Qassim University, KSA.

E-mail: docsajad@yahoo.co.in

Received: 20 July 2020 / Accepted: 26 August 2020 was conducted to study the status and patency of the shunt. A multiaxial CT scan of the abdomen and pelvis with oral and intravenous contrast (Figure 1: A-F) showed a heterogeneous liver and a patent TIPS stent communicating the porto-venous and systemic system. The portal vein was distended with multiple collater- 
als. There was neither ascites nor splenomegaly (spleen size: $26 \mathrm{~cm}$ ). The rest of the study was unremarkable. Budd-Chiari syndrome (BCS) is a very rare condition, affecting one in one million adults. BCS is named after George Budd, a British internist, and Hans Chiari, an Austrian pathologist. BCS affects younger to middleaged patients with a mean age of 40 years. The condition is caused by an occlusion of the hepatic venous outflow at any level from the small hepatic veins to the atriocaval junction ${ }^{[1]}$. The obstruction may be thrombotic or non-thrombotic. BCS is classified as primary $(75 \%)$ or secondary $(25 \%)$ depending on the origin of the obstructive lesion. If the obstruction results from endoluminal venous lesion-like thrombosis, it is termed primary BCS. However, if the cause originates from neighboring structures, such as extrinsic compression or tumor invasion, it is referred to as secondary BCS ${ }^{[2]}$.

Two of the hepatic veins must be blocked to cause clinically evident disease that presents with a classical triad of abdominal pain, ascites, and liver enlargement. The syndrome can be fulminant, acute, subacute, chronic, or asymptomatic. Acute or sub-acute variants with diffuse occlusion of the hepatic veins still have a high mortality rate and remain challenging to treat clinically. The transjugular intrahepatic portosystemic shunt (TIPS) has been used in recent times successfully and safely in selected patients of BCS as a definitive treatment or as successful bridge to liver transplant ${ }^{[3,4]}$. The most common indications for TIPS in BCS patients include progressive liver failure, refractory ascites, recurrent variceal bleeding, and diffuse hepatic vein thrombosis, and successful TIPS insertion has been found to improve hemodynamic and clinical parameters ${ }^{[5]}$.

Insertion of a TIPS involves a technique that starts with insertion of a $5 \mathrm{~F}$ multipurpose catheter into the hepatic vein. Angiography is performed, and the occlusion is identified. A puncture needle is advanced into the por- tal vein through the liver parenchyma from the inferior vena cava (IVC), and then the guide wire is placed into the portal vein (PV) through the $10 \mathrm{~F}$ sheath. Portal vein angiography is performed, and the portal vein pressure and right atrium pressure are measured to calculate the porto-systemic pressure gradient (PSG). The TIPS shunt is dilated with an angioplasty balloon of 8 or $10 \mathrm{~mm}$ in diameter, and then a covered stent with a diameter of 8 or $10 \mathrm{~mm}$ is deployed ${ }^{[6]}$. In a recent study, Qi X. documented procedure-related complications in up to $56 \%$ of cases after TIPS insertion and shunt dysfunction in $18-100 \%$ of cases. The shortand long-term prognosis of BCS-TIPS patients has been found to be excellent, with a one-year cumulative survival rate of $80-100 \%$ and a five-year cumulative survival rate of $74-78 \%{ }^{[5]}$.

\section{REFERENCES}

1. Horton, J. D. , Miguel, F. L. S. , \& Ortiz, J. A. . (2010). Buddchiari syndrome: illustrated review of current management. Liver International, 28(4), 455-466.

2. Aydinli, M. , \& Bayraktar, Y. . (2007). Budd-chiari syndrome: etiology, pathogenesis and diagnosis. World J Gastroenterol, 13(19), 2693.

3. Fox, M. A., Fox, J. A., \& Davies, M. H. (2011). Budd-Chiari syndrome--a review of the diagnosis and management. Acute Medicine, 10(1), 5-9.

4. Olliff, S. P. (2006). Transjugular intrahepatic portosystemic shunt in the management of Budd Chiari syndrome. European journal of gastroenterology \& hepatology, 18(11), 1151-1154.

5. Qi, X., Yang, M., Fan, D., \& Han, G. (2013). Transjugular intrahepatic portosystemic shunt in the treatment of Budd-Chiari syndrome: a critical review of literatures. Scandinavian journal of gastroenterology, 48(7), 771784.

6. He, F., Zhao, H., Dai, S., Wu, Y., Wang, L., Huang, H., ... \& Liu, F. (2016). Transjugular intrahepatic portosystemic shunt for Budd-Chiari syndrome with diffuse occlusion of hepatic veins. Scientific Reports, 6, 36380. 